Cite this: Soft Matter, 2014, 10, 3192

\title{
Friction of hydrogels with controlled surface roughness on solid flat substrates
}

\begin{abstract}
Shintaro Yashima, ${ }^{a}$ Natsuko Takase, ${ }^{b}$ Takayuki Kurokawa ${ }^{c}$ and Jian Ping Gong ${ }^{\star c}$
This study investigated the effect of hydrogel surface roughness on its sliding friction against a solid substrate having modestly adhesive interaction with hydrogels under small normal pressure in water. The friction test was performed between bulk polyacrylamide hydrogels of varied surface roughness and a smooth glass substrate by using a strain-controlled rheometer with parallel-plates geometry. At small pressure (normal strain 1.4-3.6\%), the flat surface gel showed a poor reproducibility in friction. In contrast, the gels with a surface roughness of 1-10 $\mu \mathrm{m}$ order showed well reproducible friction behaviors and their frictional stress was larger than that of the flat surface hydrogel. Furthermore, the flat gel showed an elasto-hydrodynamic transition while the rough gels showed a monotonous decrease of friction with velocity. The difference between the flat surface and the rough surface diminished with the increase of the normal pressure. These phenomena are associated with the different contact behaviors of these soft hydrogels in liquid, as revealed by the observation of the interface using a confocal laser microscope.
\end{abstract}

Received 15th November 2013 Accepted 28th January 2014

DOI: $10.1039 / \mathrm{c} 3 \mathrm{sm} 52883 a$

www.rsc.org/softmatter investigated. Studies on the sliding friction of various kinds of hydrogels against smooth rigid substrates as well as against the hydrogels in aqueous environment have revealed very rich and complex frictional behaviors. ${ }^{13-20}$

To reveal the role of surface roughness of living tissues in friction, studying the effect of surface roughness on friction of hydrogels in liquid is helpful. In our previous work, sliding friction of polyvinyl alcohol (PVA) hydrogels with different Young's moduli against weakly adhesive glass substrates of varied surface roughness has been studied under a large normal strain of $26 \% .^{21}$ With an increase in substrate roughness, the friction in the low velocity region increases slightly, while it decreases significantly above a critical velocity on the substrate with a roughness larger than $1 \mu \mathrm{m} .{ }^{21}$ The velocitydependence of gel friction on rough rigid surfaces is understood by the surface contact dynamics. At low velocity, the adhesive gel has enough time to dewet the substrate and form contact at the interface, so the friction increases with the roughness due to the enhanced contact area between the gel and the substrate at large normal strain. At high velocity, the gel does not have sufficient time to form complete contacts with the substrate surface asperities, so the friction decreases with the velocity. This result provides some essential ideas for designing a soft gel system with low friction over a wide velocity range, which is important in bioengineering applications where low friction is required.

It is easy to speculate that, the opposite case, i.e., the friction of soft hydrogels with surface roughness on a smooth rigid substrate, should exhibit completely different behaviors, due to the asymmetry in their elastic modulus.
${ }^{a}$ Graduate School of Life Science, Hokkaido University, Sapporo 060-0810, Japan ${ }^{b}$ School of Science, Hokkaido University, Sapporo 060-0810, Japan

${ }^{c}$ Faculty of Advanced Life Science, Hokkaido University, Sapporo 060-0810, Japan. E-mail: gong@mail.sci.hokudai.ac.jp 
In this paper, we study the sliding friction of hydrogels with varied surface roughness on a smooth solid substrate having modestly adhesive interaction with the hydrogels in water.

As the roughness effect of hydrogels will diminish under very large normal compression, we confined our study to the condition that the normal pressure is very low relative to the modulus of the gel, corresponding to a normal strain in the range of $1.4-3.6 \%$. It should be mentioned that under such low strain conditions, we usually observe poor reproducibility of friction in the case of flat surface hydrogels. Due to this reason, most of our previous studies on hydrogels friction have been performed at relatively large strains, usually above $10 \% .^{22-24}$ In this study, we revealed that the poor reproducibility is due to the water entrapment, and found out that this problem can be solved by using rough surface gels. We have found that the rough surface of hydrogels enhances the soft contact in liquid. Due to this, the friction of rough surface hydrogels is higher than that of the flat gel. The results obtained in this study give insight into the understanding of hydrogel contact in liquid, which is important not only for hydrogel friction, but also for hydrogel adhesion under water.

In this work, we used the PAAm hydrogel for the friction study. There are advantages of using the PAAm gel. (1) The PAAm gel is synthesized from a monomer solution that has low viscosity. This made it possible to replicate the shape of templates with a fine resolution; and (2) the PAAm gel can be easily labelled by copolymerizing AAm with a fluorescent monomer. This paper is arranged as follows. First, we present the frictional behavior of a chemically crosslinked neutral hydrogel, poly(acrylamide) (PAAm) gel, with varied surface roughness against a smooth glass substrate in water. PAAm gels have a higher adhesion to the glass substrate in water than PVA gels that have been used in our previous studies. ${ }^{21}$ Then we present the observation on the contact of the PAAm hydrogels to glass by using a confocal laser microscope. Based on these two experiments, we discuss the effect of roughness on the friction in terms of soft contact mechanics in liquid. Finally, we present the conclusions.

\section{Experimental}

\section{Materials}

Hydrogels. PAAm gels with various levels of surface roughness were synthesized by radical polymerization. An aqueous solution of $2 \mathrm{M}$ acrylamide (AAm, Tokyo Kasei Co., Ltd.) as the monomer, 1 mol\% $N, N^{\prime}$-methylene bisacrylamide (MBAA, Tokyo Kasei Co., Ltd) as the crosslinker, and $0.1 \mathrm{~mol} \%$ potassium peroxodisulfate (KPS, Kanto Chemical Co., Inc.) as the initiator, the latter two are respective to the monomer, was prepared first. Then, $50 \mu \mathrm{l}$ of $N, N, N^{\prime}, N^{\prime}$-tetramethylethylenediamine (TEMED; Tokyo Kasei Co., Ltd., Japan) was dropped into $20 \mathrm{ml}$ of the aqueous solution. Then, the solution was poured into a home-made reaction cell consisting of two glass plates separated with a silicone rubber spacer of $2.0 \mathrm{~mm}$ thickness. One of the glass plates is a template with programmed roughness. Polymerization was carried out at room temperature for $8 \mathrm{~h}$. After gelation, samples were immersed in a large amount of ion-exchanged water for 1 week and water was changed two times every day to remove residual chemicals.

Rough templates. In order to synthesize hydrogels with various levels of surface roughness, glass templates with programmed surface roughness (Niki Token Co., Ltd.) were used. These glass templates were obtained by sandblasting using different sizes of particles.

Rigid substrates. A glass base dish (internal diameter: $27 \mathrm{~mm}$ ) purchased from Asahi glass Co., Ltd. was used as the rigid substrate for frictional measurement and observation of the hydrogel-glass interface.

\section{Measurements}

Surface roughness of hydrogels. Surface roughness of gel samples was observed with a geometry measurement laser microscope (VK9710, Keyence Corp). This microscope performs laser scanning in the plane parallel to the sample surface $(X-Y$ planes) at various depths from the sample surface ( $Z$-axis) in a programmed range. The stepping distance on the $Z$-direction was set to $0.1 \mu \mathrm{m}$. In order to avoid drying of gel samples, the $X-Y$ range was set to $500 \mu \mathrm{m} \times 750 \mu \mathrm{m}$ at the maximum to shorten the observation time. The observation time was 5-15 minutes, approximately, depending on the scanning range. As a result, well-focused 3-D images of the surface morphology were achieved. The average roughness $\left(R_{\mathrm{a}}\right)$ was estimated as the arithmetic average of the profile ordinates within the measured section (average height). 3 identical samples, at 4 different positions from each sample, were measured. $R_{\mathrm{a}}$ was the average of 12 data from these measurements.

Elasticity of hydrogels. The Young's modulus $E$ of the gel was $130 \mathrm{kPa}$. It was measured by using a tensile-compressive tester (Tensilon, Orientec., Co.). The disc-shaped sample of smooth surface, $10 \mathrm{~mm}$ in diameter and $2.4 \mathrm{~mm}$ in thickness, was compressed with a velocity of $10 \%$ thickness $\min ^{-1}$. Using strain and nominal stress, the Young's modulus was calculated at a strain of less than $10 \%$. Modulus data were the average over 3 samples.

Contact angle to water. The contact angle to water $\theta$ of the glass substrate and hydrogels was measured with a Drop Master 300 (Kyowa Interface Science Co., Ltd.) in air at room temperature. The volume of the water droplet $(10 \mu \mathrm{l})$ was maintained constant for all the measurements in order to prevent changes in the droplet due to the gravity effect. $\theta$ of the glass substrate was $42 \pm 2^{\circ}$. $\theta$ of the PAAm gel was $21 \pm 4^{\circ}$. Each of the data was an average over 24 points. 4 samples were used and 6 places of each sample were measured.

Frictional measurement. The observation of the friction was performed using a commercially available rheometer (3ARES17A, Rheometric Scientific F. E. Ltd.), operated in a constant compressive strain mode. The disc-shaped hydrogel, which was cut into $15 \mathrm{~mm}$ in diameter, was glued on the upper plate of the rheometer using a cyanoacrylate instant adhesive agent (Toa Gosei Co., Ltd.). The thickness of hydrogels was $2.4 \mathrm{~mm}$. The frictional objective substrate, that is, the glass dish (Asahi glass CO., Ltd.), was glued onto the lower plate of the rheometer. The normal pressure $P$ was set as an experimental parameter. Prior 
to the friction measurement, a normal strain was applied, and it was increased gradually until the normal stress reached a prescribed value. After 15 min of preloading, the friction was measured in the steady rate sweep test (SRST) mode. An angular displacement was applied to the lower plate at a constant angular velocity $(\omega)$. The angular velocity $\omega$ was increased stepwise from $10^{-3}$ to $10 \mathrm{rad} \mathrm{s}{ }^{-1}$ without separating the two rotating surfaces. For each $\omega$, the measurement lasted for $40 \mathrm{~s}$, and the average torque $T$ of the last $20 \mathrm{~s}$ was adopted. No stickslip was observed. The frictional force, $F$, was calculated as $F=4 T / 3 R$, where $T$ was the frictional torque recorded during measurement and $R(=7.5 \mathrm{~mm})$ was the radius of the apparent contact area. The average frictional shear stress, $\sigma$, generated at the interface was qualified as the friction force per unit area, $\sigma=F / \pi R^{2}$. Although the sliding velocity varies along the radial direction in parallel plate geometry, we adopted the sliding velocity $v$ at the outer side of disk-shaped samples, $v=\omega R$.

After the first run, the normal strain was released and the gel sample was totally separated from the substrate for $5 \mathrm{~min}$. After that, normal strain was applied again for $15 \mathrm{~min}$, whereupon the 2 nd run of measurement was performed. 3rd run and 4 th run were performed in the same way.

Friction of gels under three normal stresses of $0.5 \mathrm{kPa}, 1.1$ $\mathrm{kPa}$, and $2.8 \mathrm{kPa}$, corresponding to normal strains of $1.4 \%$, $2.3 \%$, and $3.6 \%$, respectively, was investigated. Due to stress relaxation, the normal strain applied was higher than $P / E$. Under this normal strain range, the misalignment of parallel plates was assumed to be negligible. Fresh samples were used for each normal stress measurement.

Observation of the gel-glass interface. The observation of the gel-glass interface was performed using a confocal laser scanning microscope (T2000, Digital Eclipse C1, Nikon CO., Ltd.) under similar conditions to the frictional measurement test. Normal force $(1.1 \mathrm{kPa})$ was applied on the gel for $15 \mathrm{~min}$ in order to allow the hydrogel to reach steady state. To visualize the water layer that existed at the gel-glass interface, water containing $1.5 \mathrm{vol} \%$ of red fluorescent particles that have a diameter of $0.4 \mu \mathrm{m}$ (FR2040, Thermo Scientific Fluoro-Max Fluorescent, Thermo Fisher Scientific Inc.) was used. It was experimentally confirmed that the particle size was large enough than the mesh size $\xi$ of the hydrogels that the particles could not penetrate into the hydrogels ( $\xi$ was estimated to be $4.6 \mathrm{~nm}$ from the modulus $E$ of the gel by using $\left.E=3 k_{\mathrm{B}} T / \xi^{3}\right)$. Also, it was experimentally confirmed that no fluorescent particles were adhered to the gel surface. Both plain PAAm samples and fluorescein labelled PAAm samples were used. In the latter case, $5 \mathrm{mmol} \%$ of fluorescent monomer 4-acryloyl fluorescein relative to the AAm monomer was copolymerized in PAAm gels. This fluorescently labelled hydrogel showed green color, and it was confirmed experimentally that this fluorescent monomer does not have effect on contact and friction.

The first observation was set up to see where the water layer exists. A lens with $40 \times$ magnification and about $57 \mu \mathrm{m}$ depth of focus was used, and the scanning area was $3180 \times 3180 \mu \mathrm{m}^{2}$. The $57 \mu \mathrm{m}$ depth of focus made it possible to include the whole water layer in one image.

The second observation was set up to estimate the thickness of the water layer. A lens with $200 \times$ magnification and about
$2.6 \mu \mathrm{m}$ depth of focus was used, and the scanning area was $636.5 \times 636.5 \mu^{2}$. By using the dually fluorescent color labelled samples, images containing the $3 \mathrm{D}$ information of the water layer were obtained at varied normal pressure $P$. The $Z$-axis was set vertical to the surface of the glass substrate, and $Z=0$ was set at the glass surface where the image was dark. Scanning along the $Z$-axis was performed towards the inner plane of gels, with each $Z$-step of $\Delta=0.5 \mu \mathrm{m}$ until the position where no red particles were detectable and the whole area of the image became green gels was reached. For estimation of mean thickness of the water layers, randomly selected 4 positions were observed for each sample.

The mean water layer thickness $h$ was calculated by eqn (1).

$$
h=\Delta \sum_{Z=0}^{Z} \frac{A_{\mathrm{w}}(Z)}{A_{\mathrm{ob}}}
$$

Here, $A_{\mathrm{w}}(Z)$ is the area of water layer at depth $Z$, which was calculated from the image of red color at depth $Z$, by using Image Pro 6 (CyberMetrics Inc.). $A_{\mathrm{ob}}(=636.5 \mu \mathrm{m} \times 636.5 \mu \mathrm{m})$ is the observation area of the image. The mean $h$ shown in Fig. 7 was the average of 4 different positions. It should be mentioned that although the resolution of water layer thickness was limited by the focusing depth of the lens, which was $2.6 \mu \mathrm{m}$, the mean water layer thickness $h$ estimated from eqn (1) could be less than $2.6 \mu \mathrm{m}$, due to the averaging over the whole observation area.

\section{Results and discussion}

Fig. 1a shows the typical surface morphology of the rough gel samples (sample S2) obtained using a geometry measurement laser microscope.
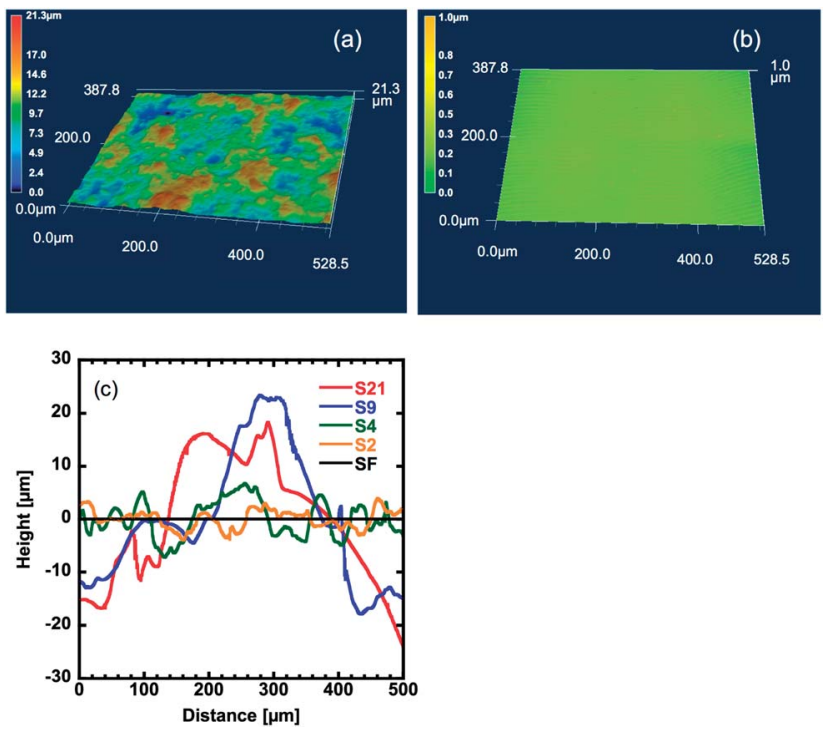

Fig. 1 ( $a$ and b) Images of surface topography of S2 (a) and SF (b) samples measured using a geometry laser microscope (Keyence VK9710). (c) Line profiles of hydrogels of various levels of surface roughness obtained from the results of the geometry laser microscope. 
The surface image of the flat sample (sample SF) is shown in Fig. 1b. The surface line profiles of samples with various levels of roughness are shown in Fig. 1c. The average roughness $\left(R_{\mathrm{a}}\right)$ is shown in Table 1.

Fig. 2 shows the frictional behavior of hydrogels with various levels of surface roughness at a normal pressure $P$ of $1.1 \mathrm{kPa}$ (strain: $2.3 \%$ ). The numbers in the graph indicate the order of measurement runs. It was found that the 1st run usually has a poor reproducibility. Therefore, we repeated several measurements and showed the results from the 2nd run. Several features were observed. (1) Frictional stress of samples with large roughness shows better reproducibility than that of the samples with small roughness. Especially, large variations in frictional stress between different runs were observed for the flat sample, especially at a low velocity region. (2) Samples with large roughness show higher frictional stress than that of samples with a flat surface. (3) In the case of rough surface gels, the frictional stress keeps decreasing at high velocity, but in the case of a flat surface gel, frictional stress decreases once and then increases with increase of sliding velocity. The result (2) is consistent with the observation of friction behavior of a wet rubber with surface roughness against a smooth rigid substrate. ${ }^{25}$ Manning et al. evaluated friction of rubber soling with surface roughness on a wet rigid surface, and showed that the friction coefficient increases with increase of surface roughness of rubber soling. ${ }^{25}$

To make a more quantitative comparison of the frictional stress between different samples, we plotted the relationships between surface roughness $\left(R_{\mathrm{a}}\right)$ and frictional stress at a

Table 1 Sample name and the mean roughness $\left(R_{\mathrm{a}}\right)$ of hydrogels used in this study

\begin{tabular}{lccccc}
\hline Sample name & S21 & S9 & S4 & S2 & SF \\
\hline$R_{\mathrm{a}}[\mu \mathrm{m}]$ & 21 & 9 & 4 & 2 & 0.04
\end{tabular}
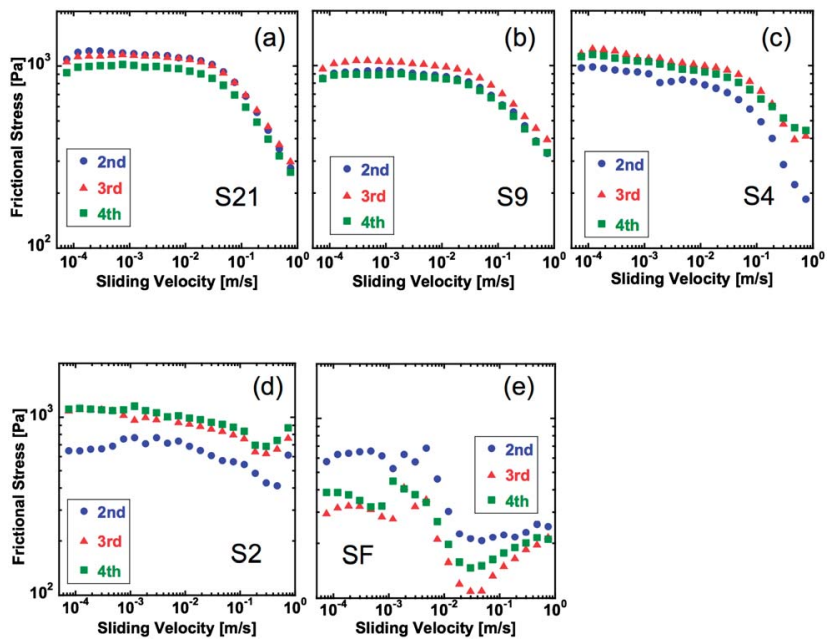

Fig. 2 Velocity dependence of dynamic frictional stress of hydrogels under a normal pressure $(P)$ of $1.1 \mathrm{kPa}$ (strain: 1.4\%). The letters in the graphs indicate sample name shown in Table 1. The data of 2 nd, 3rd, and 4 th run of the measurement are shown. constant velocity (Fig. 3). At low velocity, frictional stress of rough surface hydrogels is several times larger than that of flat gels. At a relatively high velocity of $7.5 \times 10^{-1} \mathrm{~m} \mathrm{~s}^{-1}$, however, these differences in the frictional stress vanish. No significant difference of frictional stress was found for different surface roughness in the experimental range investigated $(2.3-21.4 \mu \mathrm{m})$.

The friction behaviors of gels under normal pressures $P$ of $0.5 \mathrm{kPa}, 1.1 \mathrm{kPa}$, and $2.8 \mathrm{kPa}$ are shown in Fig. 4. Here, the friction stress was the average of 3 runs for each sample. With the increase of $P$, the frictional stress increases for both the flat sample and rough samples, while this effect of $P$ is more prominent in the case of the flat gel. As a result, the behavior of the flat sample approaches that of the rough samples at a high value of $P$. Also, the specific velocity at which the frictional force begins to decrease shifts to a high value for the flat sample, while that of rough surface gels almost does not change.

Intuitively, the surface roughness of the soft hydrogels would reduce the contact area to the solid substrate, and therefore, would reduce the friction. The opposite phenomenon observed suggests that the rough surface of the hydrogels enhances the contact of the gel with the glass substrate. To elucidate the mechanism for the roughness effect observed, we investigate the interfacial contact by carrying out the direct observation of the gel-glass interface. Fig. 5 and 6 show the images of the hydrogel-glass interface under a normal pressure of $P=1.1 \mathrm{kPa}$ for rough gels and flat gel, respectively. As the observation depth $(\sim 57 \mu \mathrm{m})$ was much larger than the roughness of the samples,

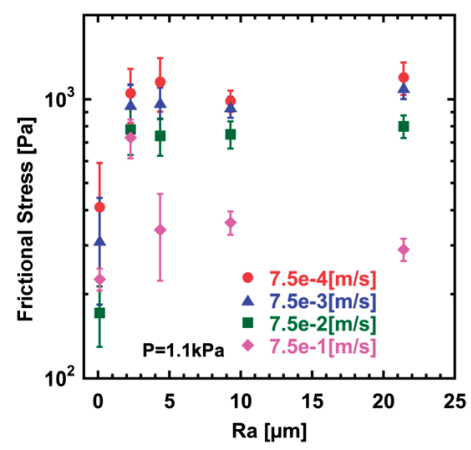

Fig. 3 Relationships between the surface roughness $\left(R_{\mathrm{a}}\right)$ of hydrogels and frictional stress under a normal pressure $(P)$ of $1.1 \mathrm{kPa}$. The sliding velocity $(v)$ is shown in the graph.
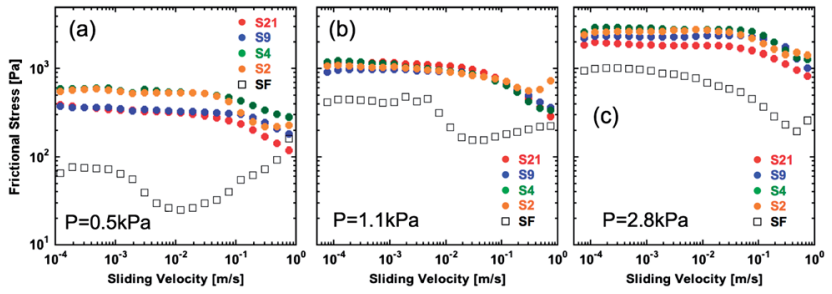

Fig. 4 Velocity dependence of dynamic frictional stress of hydrogels with varied surface roughness against a smooth glass substrate under three different normal pressures $(P)$. (a) $P=0.5 \mathrm{kPa}$, (b) $P=1.1 \mathrm{kPa}$, and (c) $P=2.8 \mathrm{kPa}$. The normal strains of (a), (b) and (c) were $1.4 \%, 2.3 \%$, and $3.6 \%$, respectively. 

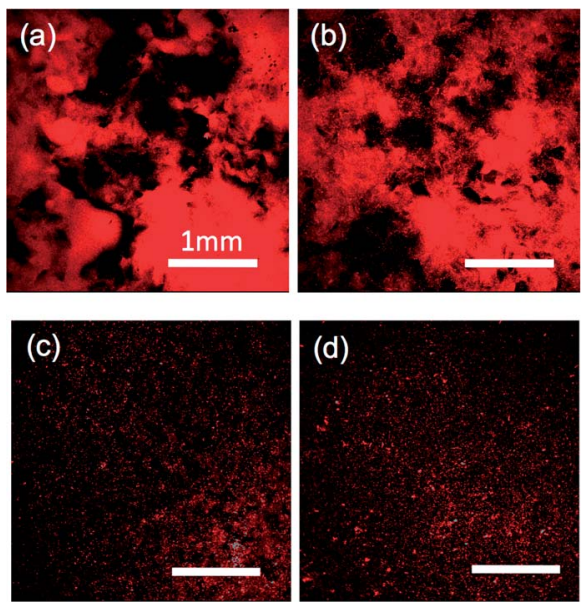

Fig. 5 Confocal laser microscopy images of the water layer labelled by red fluorescent particles at the gel-glass interface. (a) S21, (b) S9, (c) S4 and (d) S2. A normal pressure of $1.1 \mathrm{kPa}$ was applied on all the gels and kept 15 minutes before taking images to get steady state, same as the friction measurement. Magnification: 40x, depth of focus: $57 \mu \mathrm{m}$. Area: $3180 \times 3180 \mu \mathrm{m}^{2}$. Each scale bar shows $1 \mathrm{~mm}$.

the strength of the red color from the fluorescent particles is an indicator of the water layer thickness at the interface. For the gels with relatively large surface roughness $(21.4 \mu \mathrm{m}$ and $9.3 \mu \mathrm{m}$, Fig. 5a and b, respectively), one can see, distinctly, the coexistence of dark areas and bright red areas with a size much larger than $R_{\mathrm{a}}$. Dark areas mean that fluorescent particles are squeezed out and the gel forms contact with glass strongly in these regions, while the bright red areas indicate that thick liquid layers exist in these regions. These images show that gels with a relatively large surface roughness can form firm contact with glass by some of their apexes of rough surface under the normal pressure.

For the gels with intermediate surface roughness $(4.3 \mu \mathrm{m}$ and $2.3 \mu \mathrm{m}$, Fig. 5c and d, respectively), large bright areas disappear. Instead, dark areas containing small bright dots are observed homogeneously over the samples.
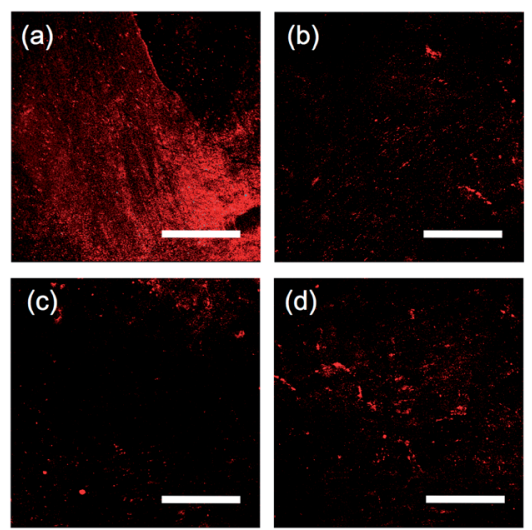

Fig. 6 Images of the water layer at the sample SF $\left(R_{\mathrm{a}}=0.04 \mu \mathrm{m}\right) \mathrm{gel}-$ glass interface at 4 different positions of one sample indicated in the illustration above the images. The contact process and the normal pressure were the same as Fig. 5. Magnification: $40 \times$, depth of focus: $57 \mu \mathrm{m}$. Area: $3180 \times 3180 \mu \mathrm{m}^{2}$. Each scale bar shows $1 \mathrm{~mm}$.
In the case of the flat gel, it is surprising to find that there is a strong position dependence of the fluorescent intensity. Fig. 6ad show images of a flat sample at 4 different positions. Both strong bright regions (Fig. 6a) and dark regions (Fig. 6b-d) with small dispersed bright dots were observed. These results indicate the entrapment of the water layer in the bright region, even for the flat sample.

To estimate the thickness of the water layer that existed at the gel-glass interface, the three-dimensional images of interface were further observed using dually labelled samples. Fig. 7a shows a typical example (sample S4) of the images at different distances from the glass surface under various normal pressures, where $0 \mathrm{kPa}$ means the applied pressure was $0 \mathrm{kPa}$ and the pressure comes from gel weight itself. With the increase of the distance from the glass surface, red area (water phase) decreases and the green area (gel phase) increases, indicating the decrease in the amount of water with the depth. At a higher normal pressure, a smaller red area is observed at the same depth, indicating the decrease of the water volume under the pressure. From three-dimensional images as shown in Fig. 7a, the average water layer thickness $h$ over the observation area at the gel-glass interface was estimated for different samples under various pressures (Fig. 7b).

The thickness $h$ shows a rapid decrease when $P$ is increased from 0 to $1.1 \mathrm{kPa}$, which suggests that the sample without applying load still did not reach the equilibrium contact after 15 min waiting. When $P>1.1 \mathrm{kPa}, h$ shows a modest decrease with the increase of $P$. The rougher the surface of the samples, the larger the $h$. The order of $h$ is $1 \sim 10 \mu \mathrm{m}$, comparable with the $R_{\mathrm{a}}$ of the gels. These results in turn validate the method used. For the flat sample (SF), $h$ is also in the order of several $\mu \mathrm{m}$.

However, if one compares the friction results (Fig. 2 and 3) and gel-glass contact results (Fig. 6 and 7), it is easy to find that they are not correlated directly. Especially, the much lower friction of the flat gel than that of the rough gels could not be explained by the thickness of water layers observed. Furthermore, even considering its partial contact with glass, it is still against the intuition that the friction of the flat hydrogel is much lower than that of the rough samples, as the rough gels only contact the substrates in their apexes and have small contact area.

In order to understand this puzzle, we first discuss the water squeezing process at the gel-glass interface. Under a normal compression $P$, the thickness of water layer $h$ between two parallel stiff plates of diameter $D$ decreases with time $t$, and it is governed by ${ }^{26}$

$$
h=D \sqrt{3 \eta / 16 P t}
$$

Here $\eta$ is the viscosity of water. Approximately, we can assume that the water layer thickness at the gel-glass interface is also governed by eqn (2). For a diameter $D=15 \mathrm{~mm}, h$ is estimated to be $297 \mathrm{~nm}, 200 \mathrm{~nm}$, and $125 \mathrm{~nm}$, respectively, for 15 min compression at pressures of $0.5 \mathrm{kPa}, 1.1 \mathrm{kPa}$, and $2.8 \mathrm{kPa}$. When the water layer thickness decreases to this 100 $\mathrm{nm}$ order, elastic dewetting occurs spontaneously, and some regions form contact while other regions do not. Also the 


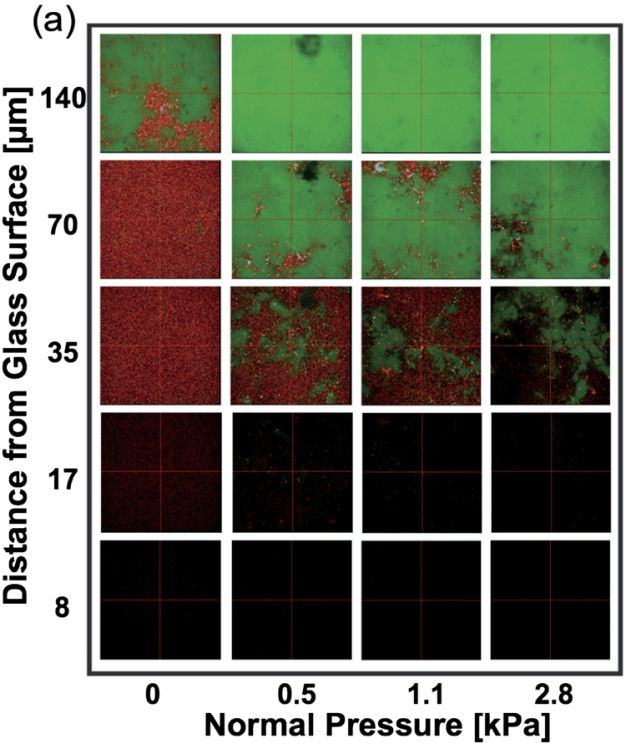

(b)

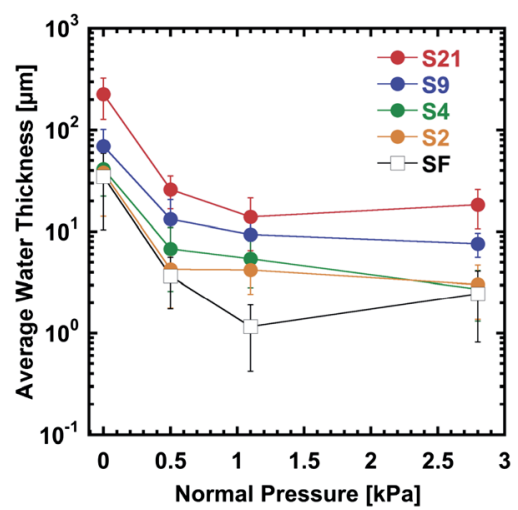

Fig. 7 (a) Images at the gel-glass interface of the dually fluorescentcolor labelled sample using a laser scanning microscope, where the hydrogel (S4) was green and the particle dispersed in water was red. The vertical axis indicates an approximate distance from the glass surface. Magnification: $200 \times$, depth of focus: $2.6 \mu \mathrm{m}$. Area: $636.5 \times$ $636.5 \mu \mathrm{m}^{2}$. (b) Relationship between the average water layer thickness $h$ at the gel-glass interface and the normal pressure $(P)$ applied on hydrogels. Error bars are standard deviations over 4 different positions.

entrapment of water, which is governed by the energy balance between the elastic deformation of the soft gel and the gel-glass adhesion, may occur. According to the elastic dewetting theory, ${ }^{27}$ the competition between the surface energy and the rubber elasticity $E$ is characterized by the elastic length $h_{0}=-S / E$. Here $S$ is the spreading constant of water at the gelglass interface. For the adhesive case, $S$ has a negative value, and $-S$ corresponds to the adhesion energy of the gel on glass. Kaneko et al. reported that $S$ of a PDMS rubber on the glass substrate in PDMS oil was $-10 \mathrm{mN} \mathrm{m}^{-1}$, and the friction of this system at low sliding velocity, in which the adhesion dominates the friction, is almost 10 times higher than that of our case. ${ }^{28}$ Therefore, we assumed $S \sim-1 \mathrm{mN} \mathrm{m}^{-1}$ for the PAAm/glass in water. Using $E=130 \mathrm{kPa}, h_{0}$ is estimated to be $\sim 10 \mathrm{~nm}$. This value is slightly larger than but very close to the mesh size of the gel $\xi$ that was estimated to be $4.6 \mathrm{~nm}$. Assuming that the elastic dewetting theory is still applicable in the present case, the thickness of the entrapped liquid $h$ is related to the radius of trapped liquid $R$ by the relationship of $h^{2} \sim R h_{0}$. For $R \sim 1 \mathrm{~mm}, h$ is about $\sim 3 \mu \mathrm{m}$. That is, when the flat gel approaches the glass substrate to form contact in liquid, a water film of large area, predicted to be several $\mu \mathrm{m}$ in thickness, can be trapped at the interface. This explains well the result shown in Fig. 7.

In the case of rough gels, the water layer between the apex of gel and the substrate is squeezed out very quickly, and no water trapping occurs. To estimate the squeezing time in the case of rough gels, we can assume that the apexes are flattened to some extent by compression, and they form flat surfaces with diameter $D$ of the same order of the apexes' diameter. For $D=100 \mu \mathrm{m}$, for example, it only takes about $30 \mathrm{~s}$ to reach $h$ of $10 \mathrm{~nm}$ even at the smallest pressure of $0.5 \mathrm{kPa}$. So, at the observation time of $15 \mathrm{~min}$, the apexes of the rough gels with surface roughness in the order of 1-100 $\mu \mathrm{m}$ form full contact with the glass.

The above discussion suggests that the water is trapped at the interface for the flat gel while water could drain freely through channels between the apexes of the surface for the rough gels. This difference in water entrapment would lead to a large difference in the local contact pressure. As schematically illustrated in Fig. 8, for the flat sample, the trapped water layer also sustains the pressure to give a homogeneous pressure distribution. So the local pressure at contact $P_{\text {local }}$ is almost the same with the nominal value $P$. Because of this, a very thin water film that could not be detected by the optical observation shown in Fig. 7 might exist at the interface and the true contact area is very small, leading to a low friction. The poor reproducibility of the friction might be related to the stochastic nucleation process of dewetting, ${ }^{27}$ which gives poor reproducibility of liquid trapping at the interface. The quick increase in the friction of the flat sample with the increase of the normal pressure, as shown in Fig. 4, may be attributed to the existence of this thin water film. At high pressure $P$, this thin water film is squeezed out and the adhesive contact area increases rapidly, leading to a remarkable increase in the frictional stress.
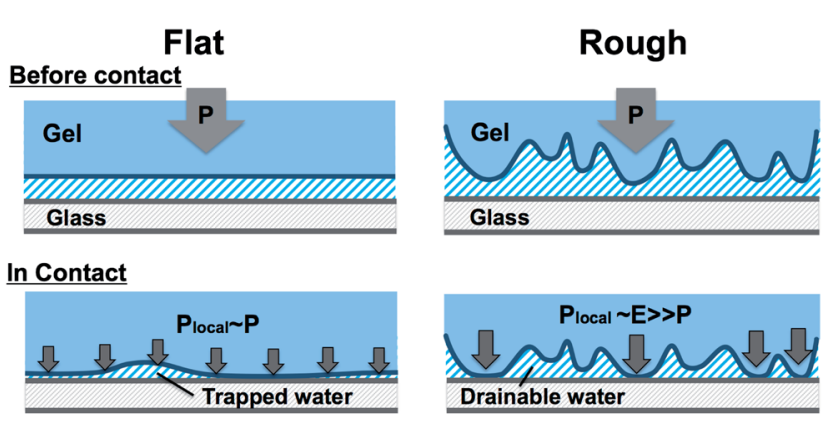

\section{At Sliding}
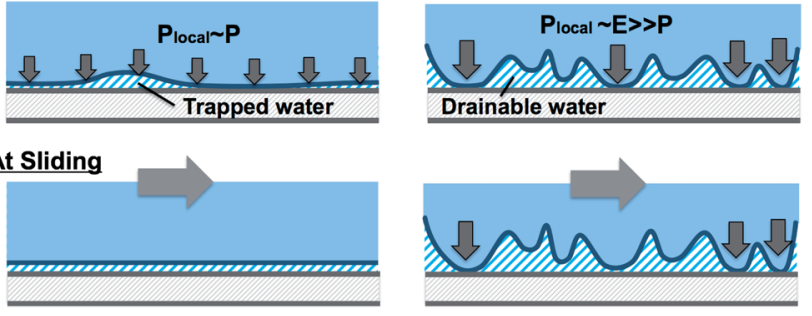

Fig. 8 Schematic illustration of the differences in the soft contact between a flat hydrogel and a rough surface hydrogel on an adhesive substrate in water. See explanation in the text. 
For rough gels, as the water at the interface does not carry the load, all the normal stress is sustained only by the apexes. So the local contact pressure $P_{\text {local }}$ is determined by the elastic modulus $E$ of the gels, much higher than the nominal value $P$. As a result, the apexes form firm adhesion to the substrate, leading to high friction. The adhesive contact area increases with $P$ by deformation of the apexes, leading to the increase in the frictional stress.

The different velocity dependence of the flat and rough gels can also be understood by the above mechanism. As the flat hydrogel takes longer time to form contact due to water entrapment, it rapidly loses the contact area with the increase of velocity. As a result, we observed a clear elasto-hydrodynamic transition at $3 \times 10^{-2} \mathrm{~m} \mathrm{~s}^{-1}$. On the other hand, the rough surface hydrogels quickly form contact with the substrate by their apexes so they maintain elastic friction even at a high sliding velocity. These velocity dependences of the friction and the elasto-hydrodynamic transition can be understood in terms of the forced wetting theory for elastic materials. ${ }^{27}$

The poor reproducibility of the friction behaviour at small pressure and the strong pressure-dependence of contact observed for the flat gel are originated from the small spreading constant of the hydrogel systems. So these behaviours are specific for hydrogels, and may not be observed in the rubber system of comparable stiffness, since the very large value of spreading constant $S$ of rubber leads to strong adhesion to the substrate even at a very small normal pressure. Furthermore, different from rubbers that are soft, adhesive but water-impermeable, hydrogels are soft, adhesive and water-permeable. So, some "additional" features coming from the fully swollen hydrogels may also play a role. For example, water could be expelled from the bulk gel under compression to reduce the adhesive strength at the contacting points. This water expelling effect, if exists, would be especially remarkable in the case of rough gels since the apexes in contact with the substrate are under large compression. However, as the friction of rough gels is found to be prominently higher than that of the flat gel, we consider that this water expelling effect, even if exists, does not play dominant role in the present case.

\section{Conclusions}

Hydrogels with a rough surface morphology showed higher and stable friction at small pressures in comparison with the hydrogels with a flat surface. While the rough gels show a monotonous decrease of friction with velocity, the flat gel shows an elasto-hydrodynamic transition within the observation velocity range. By the observation of the gel-glass interface using a confocal laser microscope, it was revealed that the flat gel traps water under pressure due to heterogeneous dewetting while rough gels provide channels to drain water. As a result, the flat gel has a small true contact with the glass substrate due to dispersion of pressure, leading to low friction at the low velocity region. In contrast to this, the apexes of rough gels form full contact with the glass surface by pressure concentration at the contact points, leading to high friction.
With the increase of sliding velocity, the contact of the flat gel to the substrate decreases rapidly, due to trapping of water. This causes a clear elasto-hydrodynamic transition at a relatively low velocity. The rough gels form contact quickly by their apexes, and thus they maintain the elastic friction even at high velocity. The poor reproducibility of the flat gel is related to the wide variation of the contact area due to water entrapment.

With the increase of normal pressure, the flat gel shows stronger friction-strengthening than that of the rough gels. As a result, the frictional behaviours of the flat gel and rough gels approach each other. The increases in the friction with the increase of pressure observed for the flat gel and rough gels are all due to the enhanced contact with the solid substrate but the mechanisms are different. The former is due to squeezing out of the water film while the latter is due to flattening of surface apexes.

These results give insight into the understanding of hydrogel contact in water, which will merit the designing of a friction system as well as adhesion system of hydrogels working at small normal pressure/strain by playing with surface roughness.

\section{Acknowledgements}

This research was financially supported by a Grant-in-Aid for Scientific Research (S) (no. 124225006) from Japan Society for the Promotion of Science (JSPS).

\section{Notes and references}

1 C. W. McCutchen, Wear, 1962, 5, 1-17.

2 J. P. Gleghorn and L. J. Bonassar, J. Biomech., 2008, 41, 19101918.

3 T. A. Schmidt and R. L. Sah, Osteoarthritis Cartilage, 2007, 15, 35-47.

4 S. Graindorge, W. Ferrandez, E. Ingham, Z. Jin, P. Twigg and J. Fisher, Proc. Inst. Mech. Eng., Part H, 2006, 220, 597-607.

5 Z. Pawlak, Z. A. Figaszewski, A. Gadomski, W. Urbaniak and A. Oloyede, Tribol. Int., 2010, 43, 1719-1725.

6 Y. M. Chen, T. Kurokawa, T. Tominaga, K. Yasuda, Y. Osada, J. P. Gong, K. Yamamoto and J. Ando, Adv. Eng. Mater., 2010, 12, B628-B636.

7 V. K. Shekhawat, M. P. Laurent, C. Muehleman and M. A. Wimmer, Osteoarthritis Cartilage, 2009, 17, 1197-1203. 8 Q. T. Nguyen, B. L. Wong, J. Chun, Y. C. Yoon, F. E. Talke and R. L. Sah, J. Biomech., 2010, 43, 1787-1793.

9 J. N. Patton and A. F. Palmer, Biomacromolecules, 2004, 6, 414-424.

10 G. Leone, M. Delfini, M. E. Di Cocco, A. Borioni and R. Barbucci, Carbohydr. Res., 2008, 343, 317-327.

11 V. M. Sardinha, L. L. Lima, W. D. Belangero, C. A. Zavaglia, V. P. Bavaresco and J. R. Gomes, Wear, 2013, 301, 218-225.

12 Y. Tao, X. Tong, Y. Zhang, J. Lai, Y. Huang, Y.-R. Jiang and B.-H. Guo, Acta Biomater., 2013, 9, 5022-5030.

13 J. Gong and Y. Osada, J. Chem. Phys., 1998, 109, 8062-8068. 14 T. Baumberger, C. Caroli and O. Ronsin, Phys. Rev. Lett., 2002, 88, 075509. 
15 T. Baumberger, C. Caroli and O. Ronsin, Eur. Phys. J. E: Soft Matter Biol. Phys., 2003, 11, 85-93.

16 Y. Ohsedo, R. Takashina, J. P. Gong and Y. Osada, Langmuir, 2004, 20, 6549-6555.

17 T. Kurokawa, T. Tominaga, Y. Katsuyama, R. Kuwabara, H. Furukawa, Y. Osada and J. P. Gong, Langmuir, 2005, 21, 8643-8648.

18 S. Oogaki, G. Kagata, T. Kurokawa, S. Kuroda, Y. Osada and J. P. Gong, Soft Matter, 2009, 5, 1879-1887.

19 A. Adjari, F. Brochard-Wyart, P.-G. de Gennes, L. Leibler, J.-L. Viovy and M. Rubinstein, Phys. A, 1994, 204, 17-39.

20 J. P. Gong, Soft Matter, 2010, 6, 2583-2590.

21 T. Tominaga, T. Kurokawa, H. Furukawa, Y. Osada and J. P. Gong, Soft Matter, 2008, 4, 1645-1652.
22 M. Du, Y. Maki, T. Tominaga, H. Furukawa, J. P. Gong, Y. Osada and Q. Zheng, Macromolecules, 2007, 40, 43134321.

23 Y. Nakano, T. Kurokawa, M. Du, J. Liu, T. Tominaga, Y. Osada and J. P. Gong, Macromolecules, 2011, 44, 8908-8915.

24 T. Tominaga, N. Takedomi, H. Biederman, H. Furukawa, Y. Osada and J. P. Gong, Soft Matter, 2008, 4, 1033-1040.

25 D. P. Manning, C. Jones, F. J. Rowland and M. Roff, Journal of Safety Research, 1998, 29, 275-283.

26 B. N. J. Perrson, Sliding Friction: Physical Principles and Applications second edition, Springer, 1998.

27 P.-G. de Gennes, F. Brochard-Wyart and D. Quéré, Capillarity and Wetting Phenomena-Drops, Bubbles, Pearls, Waves, 2003.

28 D. Kaneko, M. Oshikawa, T. Yamaguchi, J. Gong and M. Doi, J. Phys. Soc. Jpn., 2007, 76(4), 043601. 